\title{
Candida krusei form mycelia along agar surfaces towards each other and other Candida species
}

\author{
Jacob Fleischmann ${ }^{1,2^{*}}$, Corey D. Broeckling ${ }^{3}$ and Sarah Lyons ${ }^{3}$
}

\begin{abstract}
Background: Candida krusei has been known to exhibit communal interactions such as pellicle formation and crawling out of nutritional broth. We noticed another possible interaction on agar surfaces, where $C$. krusei yeast cells formed mycelia along agar surfaces toward each other. We report here the results of experiments to study this interaction.

Results: When C.krusei yeast cells are plated in parallel streaks, they form mycelia along agar surfaces toward other yeasts. They also detect the presence of Candida albicans and Candida glabrata across agar surfaces, while the latter two react neither to their own kind, nor to C. krusei. Secreted molecule(s) are likely involved as C.krusei does not react to heat killed C. krusei. Timing and rate of mycelia formation across distances suggests that mycelia start forming when a secreted molecule(s) on agar surface reaches a certain concentration. We detected farnesol, tyrosol and tryptophol molecules that may be involved with mycelial formation, on the agar surfaces between yeast streaks. Unexpectedly the amounts detected between streaks were significantly higher than would have expected from additive amounts of two streaks. All three Candida species secreted these molecules. When tested on agar surface however, none of these molecules individually or combined induced mycelia formation by C. krusei.

Conclusions: Our data confirms another communal interaction by C. krusei, manifested by formation of mycelia by yeast cells toward their own kind and other yeasts on agar surfaces. We detected secretion of farnesol, tyrosol and tryptophol by $C$. krusei but none of these molecules induced this activity on agar surface making it unlikely that they are the ones utilized by this yeast for this activity.
\end{abstract}

\section{Background}

Candida species have grown in importance as human pathogens, now being the fourth most common organisms isolated from blood cultures [18]. Candida krusei while representing a small percentage of these isolates, has become important as a pathogen for immunocompromised patients, especially because of its intrinsic resistance to some anti-fungal agents [17]. It also has been recognized to be playing a role in food fermentation [20] especially in the production of cocoa [11]. Interesting interactions among these yeasts have been described. For example, quorum sensing (QS), a synchronous

\footnotetext{
* Correspondence: jfl@ucla.edu

'David Geffen School of Medicine at UCLA, Los Angeles, CA, USA

${ }^{2}$ Research Division of GLA VA, Los Angeles, CA, USA

Full list of author information is available at the end of the article
}

expression of molecules as a function of population density, originally described in bacteria [13] has been found to be present in Candida species [1]. At high population densities Candida albicans produces farnesol which inhibits hyphal transformation, a major feature of this yeast [10]. Other alcohols with quorum sensing activities in C. albicans include tyrosol [5] and tryptophol [8]. Unusual communal behavior has also been described for C. krusei. It forms pellicles across the broth as it grows and also has the capacity as a yeast to crawl out of the nutritional broth along the inner side of tubes [19]. We have reported this phenomenon to be useful in the phenotypic identification of this organism [7]. Colonies growing on agar surface are known to eventually form mycelial elements at the periphery [16]. We now report interesting features to this mycelial formation on

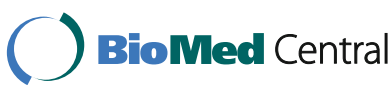

(c) The Author(s). 2017 Open Access This article is distributed under the terms of the Creative Commons Attribution 4.0 International License (http://creativecommons.org/licenses/by/4.0/), which permits unrestricted use, distribution, and reproduction in any medium, provided you give appropriate credit to the original author(s) and the source, provide a link to the Creative Commons license, and indicate if changes were made. The Creative Commons Public Domain Dedication waiver (http://creativecommons.org/publicdomain/zero/1.0/) applies to the data made available in this article, unless otherwise stated. 
agar. It appears that it senses the presence of its own kind and other Candida species across the agar surface.

\section{Methods}

Organisms and chemicals

Candida krusei ATCC 14243, Candida albicans SC5314, ATCC MYA 2876, Candida glabrata ATCC MYA 2950 and were maintained in $50 \%$ glycerol in YPD broth $(2 \%$ $\mathrm{w} / \mathrm{V}$ peptone, $1 \% \mathrm{w} / \mathrm{v}$ yeast extract, $2 \% \mathrm{w} / \mathrm{v}$ dextrose) at $-80{ }^{\circ} \mathrm{C}$. Cells were grown in YPD broth at $30{ }^{\circ} \mathrm{C}$ and maintained on Sabouraud's dextrose agar at $4{ }^{\circ} \mathrm{C}$, passaged every 4-6 weeks up to 4-5 times. Additional clinical isolates of $C$. krusei identified as such by MALDITOF, were obtained from the UCLA Clinical Microbiology Laboratory. Farnesol (F203), tyrosol (188255) and tryptophol (T90301) were all obtained from Sigma.

\section{Agar surface assay}

Unless otherwise noted organisms were lifted from agar surface with $2 \mathrm{~mm}$ sterile loop and streaked unto Sabouraud's dextrose agar in parallel (Fig. 1). Distances between streaks were as indicated in results. Any growth activity between parallel streaks is referred to as "inside" and those not in-between are referred to as "outside". Non-viable controls were yeast grown in YPD broth at $30{ }^{\circ} \mathrm{C}$, autoclaved and spun down and a loopful streaked from the pellet. Plates were incubated at $30{ }^{\circ} \mathrm{C}$ and observed daily for any mycelial formations, either with an inverted scope or a regular microscope at $4 x$ and $10 x$ magnification. Pictures were obtained with a Canon Vixia HF S30 camera adapted to microscope eyepiece tube with a MM99 adapter S/N:5343 (Martin

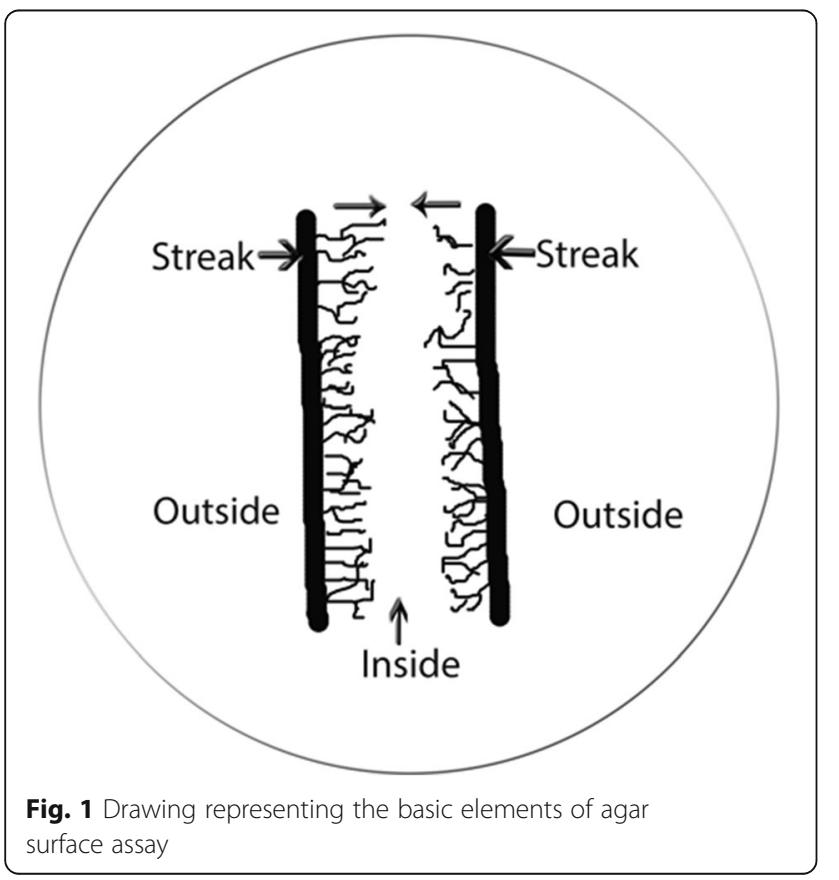

Microscope Co). To look at possible effect of number of yeasts streaked, a single colony was grown overnight in YPD with shaking at $30{ }^{\circ} \mathrm{C}$ and a loopful from this mixture or from a 1:10 dilution into fresh YPD broth were streaked.

\section{Gas Chromatography Mass Spectrometry (GC-MS)}

Organisms were streaked $10 \mathrm{~mm}$ apart and grown at $30{ }^{\circ} \mathrm{C}$ for three days. Gel pieces were punched out with sterile pipette tips that were shortened with sterile blades to give the same sized opening. They were obtained from either between parallel streaks (Fig. 1. - inside, about $5 \mathrm{~mm}$ from streaks) or outside of parallel streaks (Fig. 1. - outside, about $5 \mathrm{~mm}$ from streak) under inverted microscope visualization to avoid organisms. They were immediately frozen at $-20{ }^{\circ} \mathrm{C}$ pending analysis for secreted molecules. Pieces from agar without streaked organisms were controls.

Gel pieces were placed into $2.0 \mathrm{~mL}$ tubes, and $1 \mathrm{~mL}$ of $80 \%$ methanol was added to the plug, containing $1 \mu \mathrm{g}$ of 13C Glucose (internal standard). For the agar only sample, it was cut into pieces of similar size to the remaining samples. The sample was extracted in a sonicating water bath for $15 \mathrm{~min}$, then vortexed at high speed and room temperature for $15 \mathrm{~min}$. This process was repeated 4 times to ensure quantitative extraction. The sample was then centrifuged at $14,000 \times \mathrm{g}$ at $4{ }^{\circ} \mathrm{C}$, and $950 \mu \mathrm{L}$ of the supernatant was transferred to a new tube. The solvent was removed under nitrogen, the extracted small molecules were derivatized in a two step derivatization process. Methoximation was performed to stabilizing carbonyl functional groups using $20 \mathrm{mg} / \mathrm{mL}$ methoxyamine hydrochloride in pyridine, incubating at $37{ }^{\circ} \mathrm{C}$ for $45 \mathrm{~min}$, with intermittent sonication and vortexing. Subsequently, $50 \mu \mathrm{L}$ of MSTFA $+1 \%$ TMCS was added to trimethylsilylate amine, carboxylic acid, and hydroxyl functional groups of the metabolites. Standard $1 \mathrm{mg} / \mathrm{mL}$ stock solutions were prepared in methanol, and a dilution series was made from 0.1 to $0.0001 \mathrm{mg} / \mathrm{mL}$. Each curve contained $13 \mathrm{C}$ glucose (internal standard).

\section{GC-MS data acquisition}

Metabolites were detected using a Trace GC Ultra coupled to a Thermo ISQ mass spectrometer (Thermo Scientific). Samples were injected in a 1:10 split ratio twice in discrete randomized blocks. Separation occurred using a $30 \mathrm{~m}$ TG-5MS column (Thermo Scientific, $0.25 \mathrm{~mm}$ i.d., $0.25 \mu \mathrm{m}$ film thickness) with a $1.2 \mathrm{~mL} / \mathrm{min}$ helium gas flow rate, and the program consisted of $80{ }^{\circ} \mathrm{C}$ for $30 \mathrm{~s}$, a ramp of $15{ }^{\circ} \mathrm{C}$ per min to $330{ }^{\circ} \mathrm{C}$, and an 8 min hold. Masses between 50 and $650 \mathrm{~m} / \mathrm{z}$ were scanned at 5 scans $/ \mathrm{sec}$ after electron impact ionization. 


\section{Data processing}

All quantitative data were processed using Thermo XCalibur Quan. Selected ions were chosen for each target, and peak areas were extracted. These areas were calibrated to concentration using the dose-response calibration curve as described above.

\section{Agar assay testing with alcohols}

Stock solutions of $10 \mathrm{mg} / \mathrm{ml}$ for farnesol, tyrosol and tryptophol were prepared in ethanol. Stock solutions were serially diluted into fresh YPD broth at 1:10 ratios, and $50 \mu \mathrm{L}$ of each concentration was spread on part Sabouraud's dextrose agar surface and allowed to get absorbed. C. krusei were streaked starting from alcohol free surface (no alcohol control) continued over the alcohol containing surface. For some experiments the alcohols were diluted into melted SBA to a final concentration of $1 \mathrm{mg} / \mathrm{ml}$ and allowed to solidify and were streaked with organisms. For controls, single and parallel streaks were placed on alcohol-free SBA. Plates were incubated at $37{ }^{\circ} \mathrm{C}$ and observed daily for mycelia formations.

\section{Results}

\section{Agar surface assays with C. krusei vs C. krusei}

As mentioned previously, C. krusei has the capacity to crawl out of incubation solution [19]. Geotropism, the capacity to respond either toward or away from gravitational force has been described for fungi [14], thus we wondered if this capacity to crawl out of solution had such a basis. To see if mycelial formation will respond similarly, we streaked out yeast cells parallel to each other on Sabouraud's dextrose agar (SDA) as illustrated on Fig. 1 and maintained the Petri dish sideways at $30^{\circ}$ $\mathrm{C}$ with the streaks being horizontal one over the other. What we saw was unexpected, as after several days we observed mycelia forming along sides of streaks facing each other and not the outer sides (Fig. 1). This suggested that the organisms were "sensing" each other across the agar surface between the streaks. All subsequent assays were done on agar plates incubated in the usual horizontal fashion. A single streak of $C$. krusei will take 5 or more days to form any mycelia at $30{ }^{\circ} \mathrm{C}$ on SDA surface (Fig. 2). When streaked in parallel, it consistently forms mycelia earlier than that on the sides facing each other, while the outer sides will take as long as single streaks. An example of this can be seen in Fig. 3, as after 2 days (a) mycelia can be seen forming on the facing inner surfaces and by the third day (b) the space is fully overgrown by mycelia. After three days, the outer surfaces (c, d) still show no evidence of mycelial growth. We have repeated this assay over 30 times with Candida krusei ATCC 14243 and it consistently behaved the same way each time. In addition, five separate clinical isolates of $C$. krusei behaved the same way.

That indeed they are reacting to other viable yeast cell can be seen in Fig. 3e. When viable yeast were streaked against non-viable ones (lighter viable, darker nonviable) no mycelia are seen after three days. The distance between streaks was $5 \mathrm{~mm}$, but we saw this also in many assays where the distance was $10 \mathrm{~mm}$. Distance between streaks did indeed make a difference as to the time it took for mycelia to appear and this is summarized on Table 1. In one experiment (data not shown), comparing one loop streaks from solution with yeast grown overnight and a 1:10 dilution of this solution, we found a more robust mycelia formation from the higher number of yeast streaked, as it related to timing and distance between streaks.

\section{Agar surface assays with C. krusei vs. other Candida species}

To see if this capacity to detect other yeast is specific for C. krusei or it can also sense other Candida species, we chose $C$. albicans a robust germinator and C. glabrata not known to germinate under usual growth conditions though a pseudohyphae-like growth when starved [6] or when exposed to macrophages have been described for it [4]. Figure 4 represents the results with C. krusei vs. C. albicans and C. glabrata. At a distance of $5 \mathrm{~mm}$ between

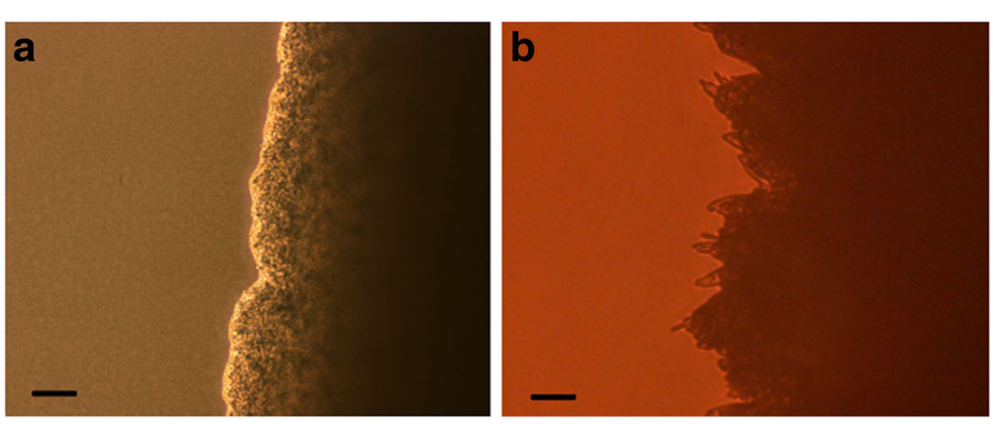

Fig. 2 Single streak of C. krusei on Sabouraud's dextrose agar incubated at $30^{\circ} \mathrm{C}$, a - after 3 days, $\mathbf{b}$ - after 5 days. For both, only one side is shown. Even at 5 days, early mycelia can be seen only in relatively small numbers. Scale bars $(S B)=100 \mu \mathrm{m}$ 

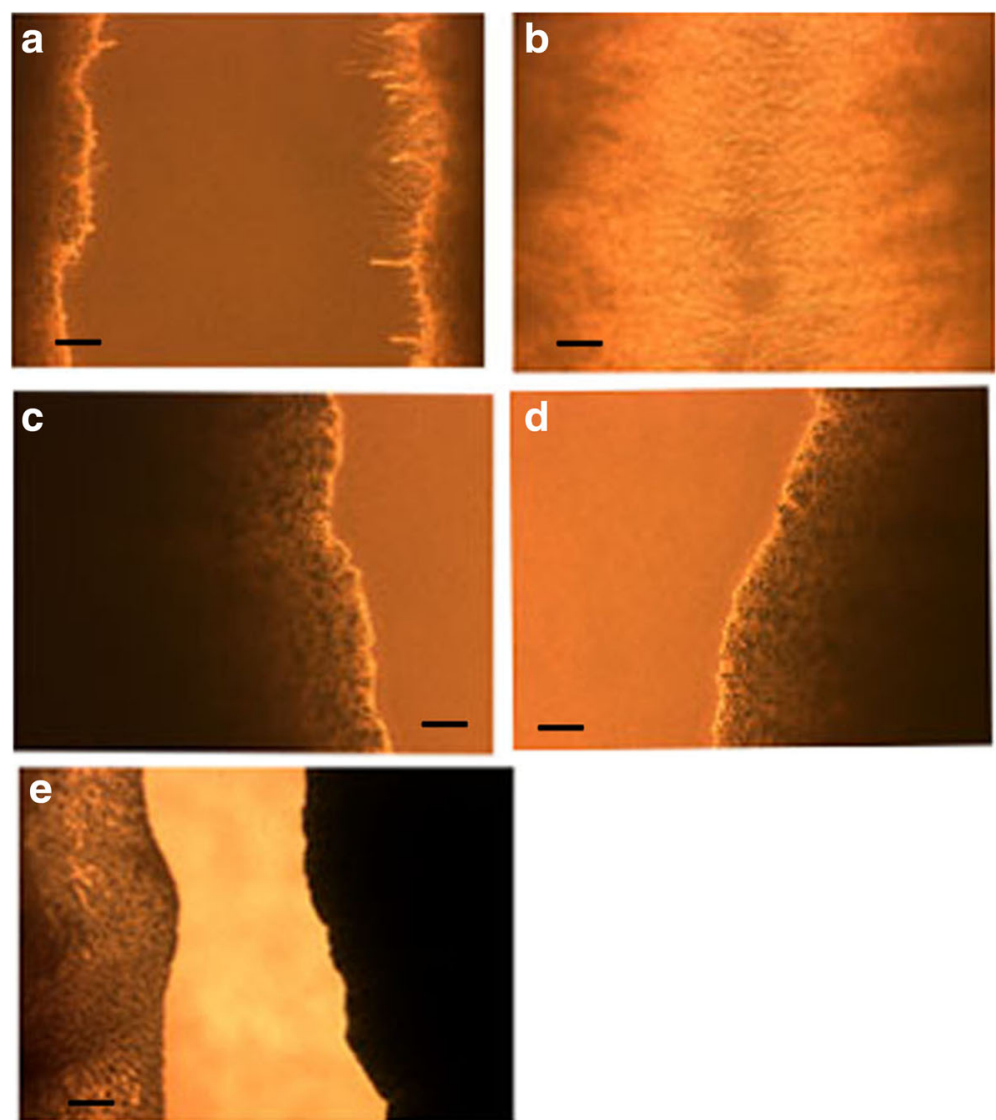

Fig. 3 Parallel streaks of C. krusei $5 \mathrm{~mm}$ apart on Sabouraud's dextrose agar incubated at $30^{\circ} \mathrm{C}$. $\mathbf{a}$ - after 2 days some early mycelia. $\mathbf{b}-$ after 3 days heavy inside mycelial rowth. $\mathbf{c}$ and $\mathbf{d}$ - outer sides of both streaks after 3 days show no outside mycelial growth. Inside and outside refer to setup shown on Fig. 1. e - Parallel streaks of C. krusei, viable (left side) vs. heat killed (right side) after 3 days, no mycelia are formed. $S B=100 \mu \mathrm{m}$

streaks by the third day (a) C. krusei vs. C. krusei show robust mycelia between them. In contrast, $C$. albicans vs. C. albicans for the same length of time (b) both inner sides show no mycelia (only one shown). In sharp contrast (c) C. krusei vs. C. albicans by day three, C. krusei is showing significant mycelia while $C$. albicans (left side of c) shows no mycelia. Similar results are seen with $C$. krusei vs. C. glabrata. On C. glabrata vs. C. glabrata (d - only one side shown) no mycelia are seen, while $C$.

Table 1 Effects of distance and time on mycelia formation by C. krusei

\begin{tabular}{llll}
\hline Distance between streaks & \multicolumn{3}{l}{ Mycelia growth after } \\
\cline { 2 - 4 } & $24 \mathrm{~h}$ & $48 \mathrm{~h}$ & $72 \mathrm{~h}$ \\
\hline $0.5 \mathrm{~cm}$ & 0 & ++ & ++ \\
$1.0 \mathrm{~cm}$ & 0 & + & ++ \\
$1.5 \mathrm{~cm}$ & 0 & 0 & + \\
\hline $0=\mathrm{no} m y c e l a$
\end{tabular}

$0=$ no mycelia

$+=$ mycelia present over less than half of streak

$++=$ mycelia present over more than half of streak

$n=2$ krusei vs. C. glabrata (e) C. krusei is germinating while C. glabrata (left side of e) is not. These results are summarized on Table 2.

\section{Analysis for secreted molecules on agar surface}

Without focusing on specific target molecules, GC-MS analysis detected a very high number of molecules on agar surface by C. krusei, making it impractical to identify the one or ones involved in this sensing activity. As our surface assays suggested that C. krusei reacted to something produced by $C$. albicans, we chose three molecules well established to be secreted by $C$. albicans. The three we looked at are farnesol, tyrosol and tryptophol. The quantitative results for all three are depicted in Fig. 5. All three yeasts produced all three alcohols with some variations. Tyrosol was the most produced by C. krusei and C. glabrata significantly more than farnesol, while $C$. albicans produced them in nearly equal amounts. The least produced by all three was tryptophol. Consistent for all three alcohols was the finding that much more could be detected between the streaks 

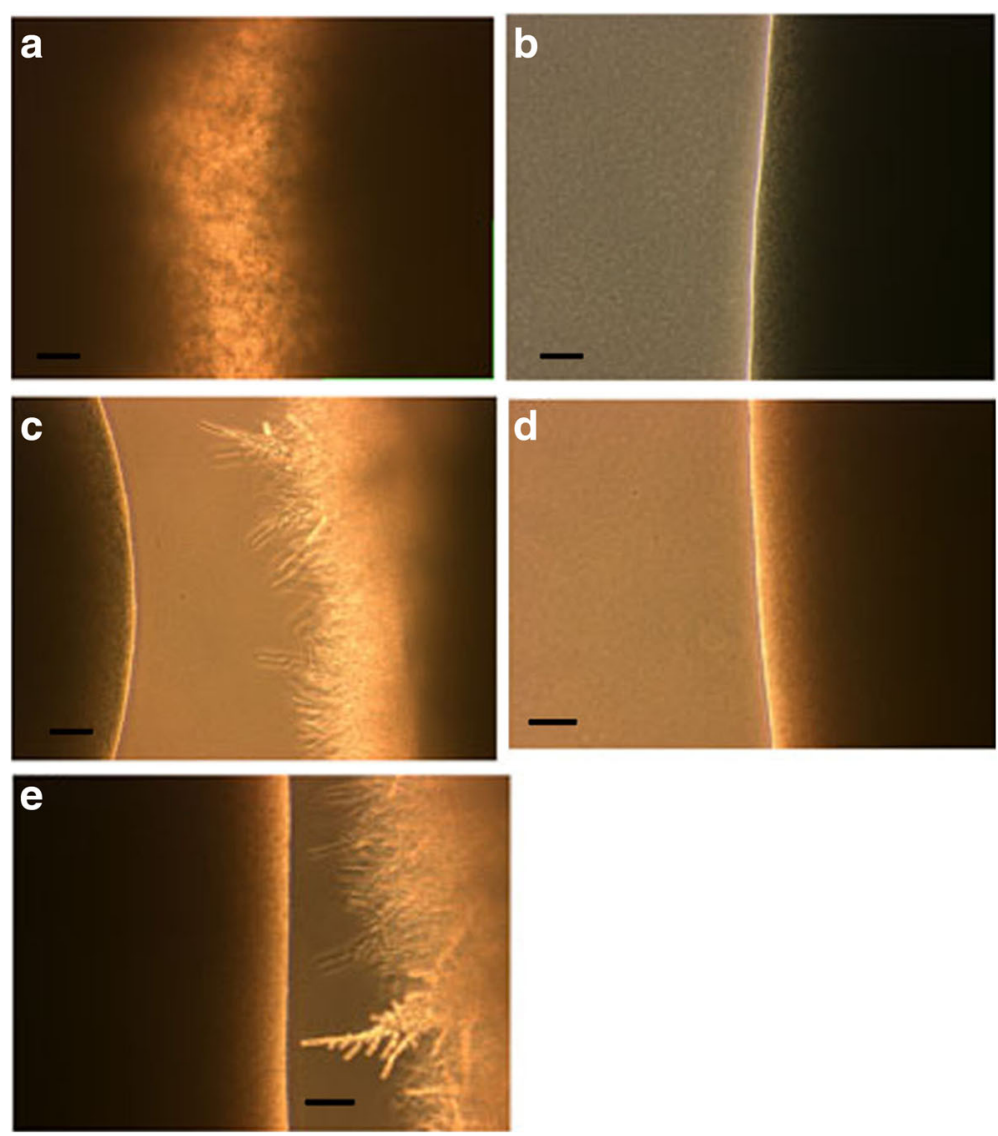

Fig. 4 Parallel streaks $5 \mathrm{~mm}$ apart, of C. krusei vs. C. albicans or C. glabrata. a - C. krusei vs. C. krusei after 3 days with robust mycelial growth as control. b - C. albicans vs. C. albicans after 3 days no mycelia are formed (only one of the inner sides shown). c - C. albicans (left) vs. C. krusei (right) showing no mycelial growth by C. albicans and robust mycelial growth by C. krusei after 3 days. d - C.glabrata vs. C. glabrata after 3 days no mycelia are formed (only one of the inner sides shown). e - C. glabrata (left) vs. C. krusei (right) showing no mycelial growth by C. glabrata and robust mycelial growth by C. krusei after 3 days. $S B=100 \mu \mathrm{m}$

Table 2 Summary of mycelia formation between streaks of pairs of Candida species

\begin{tabular}{ll}
\hline Organisms $^{\mathrm{a}}$ & $72 \mathrm{~h}$ \\
\hline C. krusei & ++ \\
C. krusei & ++ \\
C. albicans & 0 \\
C. albicans & 0 \\
C. glabrata & 0 \\
C. glabrata & 0 \\
C. krusei & ++ \\
C. albicans & 0 \\
C. krusei & ++ \\
C. glabrata & 0 \\
\hline
\end{tabular}

$++=$ more than $50 \%$ of the streak forming mycelia for the specific yeast $0=$ no mycelia formed by yeast

${ }^{\text {a }}$ Pairs represent candida species streaked parallel to each other
(10 $\mathrm{mm}$ apart) than outside of them, by day three. No detection on agar alone clearly confirms yeast secretions.

\section{Agar assay with alcohols}

To see these if these described quorum sensing alcohols played a role in mycelial formation, they were tested individually, in combinations of two and three and at various concentrations. At no time did we observe any mycelia formation on a single streak any sooner than the single control. The parallel streaks on agar alone formed mycelia as usual.

\section{Discussion}

Our observation of C. krusei sensing each other on agar surface, points to another interesting phenotypic behavior by this yeast in addition to pellicle formation on broth surface and crawling out of broth. The fact that differences in distances between streaks affected the time of appearance in mycelia is consistent with a 


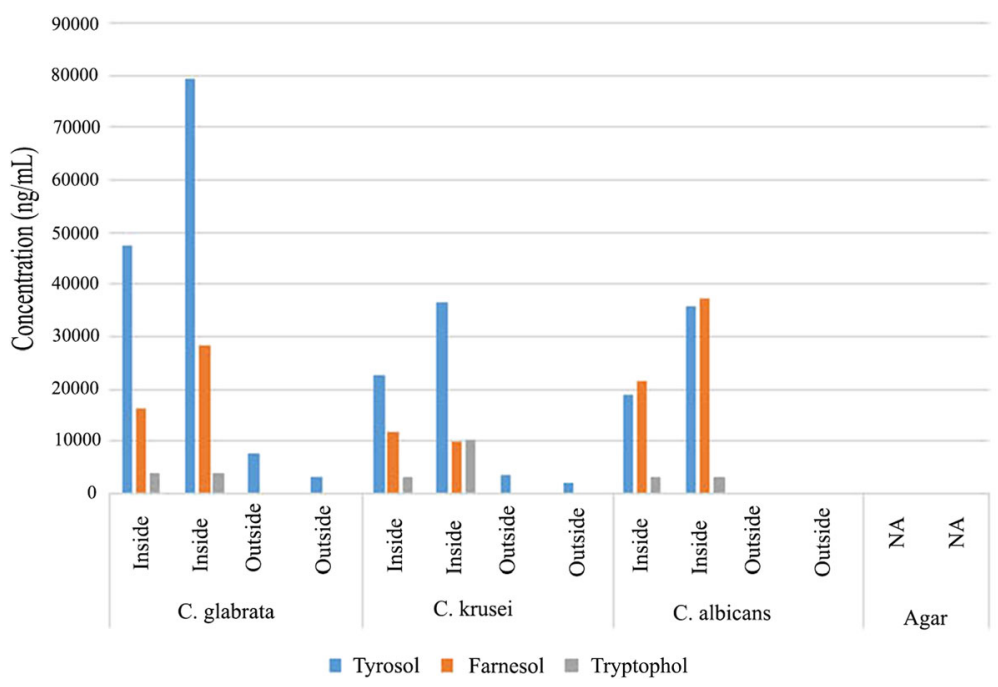

Fig. 5 Quantities of tyrosol, farnesol and tryptophol produced by Candida species on agar surface as measured by GM-CS. Distance between streaks was $10 \mathrm{~mm}$ and inside and outside refer to setup shown on Fig. 1 (see text for more details). Agar without any yeast growing on it resulted in none of the 3 alcohols detected

molecule (or molecules) secreted by the yeast along the agar surface that induces mycelial growth when it reaches a certain concentration. Our data also shows that this is not limited to self-recognition as it also reacts to C. albicans and C. glabrata. At least among these three yeasts, it seems to be unique to $C$. krusei as neither of the other two react to themselves nor to C. krusei. Mycelial formation by C. krusei appears to be specific for agar surface as we have not observed any such activity either in YPD broth or in serum, the latter being a potent inducer of hyphal formation for C. albicans [3]. Thigmotropism, growth along surfaces in response to contact has been described for C. albicans [12] and may play a role in this behavior by $C$. krusei but clearly our data shows it to be focusing at specific targets on the agar surface, which requires more than contact stimuli. Not unexpectedly, unfocused GC-MS disclosed many molecules, some of which surely originated from the agar. If one of the three molecules we detected did mediate this process, it is interesting that all three yeasts are producing it, yet only one of them, namely C. krusei is reacting to it. Other possible less specific triggers include $\mathrm{pH}$ changes at agar surface and depletion of nutrients causing C. krusei to form mycelia to seek nutrients.

Among the alcohols we looked at, farnesol actually inhibited yeast-to-mycelium transition in C. albicans [10]. Once the cells were already committed to mycelial formation however, it no longer inhibited them [15]. On the other hand tyrosol stimulates filamentation [2]. The role for tryptophol in filamentation remains unclear [1]. Our GC-MS data clearly establishes the secretion of these alcohols on agar surface by all three yeasts, but we could not demonstrate any role for them inducing filamentation across agar surface. Another yeast, important in food production Debaryomyces hansenii similarly produced tyrosol with an effect on some functions such as adhesion and slide motility but had no effect on pseudohyphal growth [9].

The one unexpected and interesting finding regarding the alcohol production by these yeasts is the quantitative difference between agar surfaces between streaks versus those outside (Fig. 5). Assuming no additional stimuli, a streak of cells would be expected to produce these molecules and secrete them along both sides onto agar surfaces at the same rate. The samples obtained were five millimeters from the streak outside and halfway between streaks which is also five millimeters from each streak. Thus if these streaks are indeed secreting these alcohols at an equal rate we would expect the amount measured inside to be additive and to be about double the amount measured on the outside. What we see is quite different. For example, for C. albicans, neither farnesol nor tyrosol could be detected outside while in between streaks significant amounts were produced. For C. krusei and C. glabrata the predominant molecule produced is tyrosol. While some could be detected outside, the ratio of inside production to outside is well above 2:1. It appears that alcohol production between streaks is boosted in a synergistic fashion. This appears to mirror mycelial formation (Fig. 2) as by the second day mycelial formation are clearly visible on the inner side of the streaks (a) and robustly by the third day (b). Yet on the outer sides of the streaks, no mycelial formations are present after the third day and usually we would see some starting to develop only after five days. One possibility is that mycelia may be the more efficient producers of these alcohols 
and therefore their production is a result of the inner robust mycelia formation and not happening parallel to it.

\section{Conclusions}

That C. krusei reacts to itself and C. albicans and C. glabrata across agar surfaces, while these two do not react to themselves or C. krusei is an unexpected finding. Since this phenomenon is manifested by mycelial formation, identifying the underlying mechanism for it should add significantly to our understanding of hyphal transformation. While we found production of farnesol, tyrosol and tryptophol by all three yeasts on agar surface, these molecules did not mediate this response. However their production exhibits an interesting quantitative pattern, accumulating to more than additive higher concentrations between parallel streaks than outside them.

\section{Acknowledgements}

We would like to thank Miguel Rocha for the art work of Fig. 1.

\section{Funding}

Elias, Genevieve and Giorgiana Atol Charitable Trust

\section{Availability of data and materials}

All data are included in the manuscript

\section{Authors' contributions}

JF - designed and carried out the experiments involving yeast behavior and wrote the manuscript. CDB - designed and carried out mass spec experiments, and assisted with the manuscript. SL - carried out mass spec experiments. All authors read and approved the final manuscript.

\section{Competing interests}

The authors declare that they have no competing interests.

\section{Consent for publication}

Not applicable

\section{Ethics approval and consent to participate}

Not applicable

\section{Publisher's Note}

Springer Nature remains neutral with regard to jurisdictional claims in published maps and institutional affiliations.

\section{Author details}

${ }^{1}$ David Geffen School of Medicine at UCLA, Los Angeles, CA, USA. ${ }^{2}$ Research Division of GLA VA, Los Angeles, CA, USA. ${ }^{3}$ Proteomics and Metabolomics Facility, Colorado State University, Fort Collins, CO, USA

Received: 24 November 2016 Accepted: 4 March 2017

Published online: 11 March 2017

\section{References}

1. Albuoquerque $P$, Casadevall A. Quorum sensing in fungi - a review. Med Mycol. 2012:50:337-45

2. Alem MA, Oteef MD, Flowers TH, Douglas LJ. Production of tyrosol by Candida albicans biofi Ims and its role in quorum sensing and biofilm development. Eukaryot Cell. 2006:5:1770-9.

3. Andleigh HS. Rapid identification of Candida albicans. Mycopathologia. 1964:23:81-4.

4. Brunke S, Seider K, Fischer D, Jacobsen ID, Kasper L, Jablonowski N, Wartenberg A, Bader O, Enache-Angoulvant A, Schaller M, d'Enfert C, Hube B. One small step for a yeast - microevolution within macrophages renders Candida glabrata hypervirulent Due to a single point mutation. PLoS Pathog. 2014;10(10):e1004478. doi:10.1371/journal.ppat.1004478.
5. Chen H, Fujita M, Feng Q, Clardy J, Fink GR. Tyrosol is a quorumsensing molecule in Candida albicans. Proc Natl Acad Sci U S A. 2004;101:5048-52.

6. Csank C, Haynes K. Candida glabrata displays pseudohyphal growth. FEMS Microbiol Lett. 2000;189:115-20.

7. Fleischmann J, Sripuntanagoon EM. Pellicle associated adherence film above incubation broth surface - an inexpensive adjunct to recognizing Candida krusei in the laboratory. BMC Res Notes. 2011;4:74. doi:10.1186/ 1756-0500-4-74.

8. Ghosh S, Kebaara BW, Atkin AL, Nickerson KW. Regulation of aromatic alcohol production in Candida albicans. Appl Environ Microbiol. 2008;74:7211-8.

9. Gori K, Knudsen PB, Nielsen KF, Arneborg N, Jespersen L. Alcohol-based quorum sensing plays a role in adhesion and sliding motility of the yeast Debaryomyces hansenii. FEMS Yeast Res. 2011;11:643-52.

10. Hornby JM, Jensen EC, Lisec AD, Tasto JJ, Jahnke B, Shoemaker R, Dussault $P$, Nickerson KW. Quorum sensing in the dimorphic fungus Candida albicans is mediated by farnesol. Appl Environ Microbiol. 2001;67:2982-92.

11. Jesperson L, Nielsen DS, Honholt S, Jakobsen M. Occurrence and diversity of yeasts involved in fermentation of West African cocoa beans. FEMS Yeast Res. 2005;5:441-53.

12. Kumamoto CA. Molecular mechanisms of mechanosensing and their roles in fungal contact sensing. Nat Rev Microbiol. 2008;6:667-73.

13. Miller MB, Bassler BL. Quorum sensing in bacteria. Annu Rev Microbiol. 2001; 55:165-99.

14. Moore D. Graviresponses in fungi. Adv Space Res. 1996:17:73-82.

15. Mosel DD, Dumitru R, Hornby JM, Atkin AL, Nickerson KW. Farnesol concentrations required to block germ tube formation in Candida albicans in the presence and absence of serum. Appl Environ Microbiol. 2005;71:4938-40.

16. Odds FC, Merson-Davies LA. Colony variations in Candida species. Mycoses. 1989:32:275-82

17. Pfaller MA, Andes DR, Diekema DJ, Horn DL, Reboli AC, Rotstein C, Franks B, Azie NE. Species of Candida in 2,496 patients: data from the Prospective Antifungal Therapy (PATH) registry 2004-2008. PLoS One. 2014;9:e101510. doi:10.1371/journal.pone.0101510.

18. Pfaller M, Neofytos D, Diekema D, Azie N, Meier-Kriesche HU, Quan SP, Horn D. Epidemiology and outcomes of candidemia in 3648 patients: data from the Prospective Antifungal Therapy (PATH Alliance ${ }^{\oplus}$ ) registry, 2004-2008. Diagn Microbiol Infect Dis. 2012;74:323-31.

19. Rao SD, Wavare S, Patil S. Onycholysis caused by Candida krusei. Indian J Med Microbiol. 2004;22:258-9.

20. Tamang JP, Watanabe K, Holzapfel WH. Review: diversity of microorganisms in global fermented foods and beverages. Front Microbiol. 2016;7:1-28.
Submit your next manuscript to BioMed Central and we will help you at every step:

- We accept pre-submission inquiries

- Our selector tool helps you to find the most relevant journal

- We provide round the clock customer support

- Convenient online submission

- Thorough peer review

- Inclusion in PubMed and all major indexing services

- Maximum visibility for your research

Submit your manuscript at www.biomedcentral.com/submit
Biomed Central 